\title{
Intravenous Immunoglobulin Therapy in Patients With Painful Idiopathic Small Fiber Neuropathy
}

Margot Geerts, MSc, * Bianca T.A. de Greef, MD, PhD,* Maurice Sopacua, MD, Sander M.J. van Kuijk, PhD, Janneke G.J. Hoeijmakers, MD, PhD, Catharina G. Faber, MD, PhD, and Ingemar S.J. Merkies, MD, PhD

Neurology ${ }^{\circledR}$ 2021;96:e2534-e2545. doi:10.1212/WNL.0000000000011919

\section{Abstract}

\section{Objective}

This is the first double-blind randomized controlled trial evaluating the efficacy and safety of IV immunoglobulin (IVIG) vs placebo in patients with idiopathic small fiber neuropathy (I-SFN).

\section{Methods}

Between July 2016 and November 2018, 60 Dutch patients with skin biopsy-proven I-SFN randomly received a starting dose of IVIG ( $2 \mathrm{~g} / \mathrm{kg}$ body weight) or matching placebo ( $0.9 \%$ saline). Subsequently, 3 additional infusions of IVIG $(1 \mathrm{~g} / \mathrm{kg})$ or placebo were administered at 3-week intervals. The primary outcome was a 1-point change in Pain Intensity Numerical Rating Scale score at 12 weeks compared to baseline.

\section{Results}

Thirty patients received IVIG, and 30 received placebo. In both groups, 29 patients completed the trial. In $40 \%$ of patients receiving IVIG, the mean average pain was decreased by at least 1 point compared to $30 \%$ of the patients receiving placebo ( $p=0.588$, odds ratio $1.56,95 \%$ confidence interval $0.53-4.53$ ). No significant differences were found on any of the other prespecified outcomes, including general well-being, autonomic symptoms, and overall functioning and disability.

\section{Conclusions}

This randomized controlled trial showed that IVIG treatment had no significant effect on pain in patients with painful I-SFN.

\section{Trial Registration Information}

ClinicalTrials.gov Identifier: NCT02637700, EudraCT 2015-002624-31.

\section{Classification of Evidence}

This study provides Class I evidence that for patients with painful I-SFN, IVIG did not significantly reduce pain compared to placebo.

\author{
Correspondence \\ Dr. Faber \\ c.faber@mumc.nl
}

\section{RELATED ARTICLE}

Editorial

IVIG and Small Fiber

Neuropathy: The Ongoing

Search for Evidence

Page 929

\section{MORE ONLINE}

$\rightarrow$ Class of Evidence

Criteria for rating

therapeutic and diagnostic studies

NPub.org/coe

\footnotetext{
*These authors contributed equally to this work.

From the Department of Neurology (M.G., B.T.A.d.G., M.S., J.G.J.H., C.G.F., I.S.J.M.), School of Mental Health and Neuroscience, and Department of Clinical Epidemiology and Medical Technology Assessment (S.M.J.v.K.), Maastricht University Medical Center+; Department of Rehabilitation Adelante/Maastricht University Medical Center+ (M.S.), the Netherlands; and Department of Neurology (I.S.J.M.), Curaçao Medical Center, Willemstad, Curaçao.

Go to Neurology.org/N for full disclosures. Funding information and disclosures deemed relevant by the authors, if any, are provided at the end of the article. 


\section{Glossary}

DSIS = daily sleep interference scale; I-SFN = idiopathic SFN; ITT = intention-to-treat; NPS = Neuropathic Pain Scale; PGIC = patients' global impression of change; PI-NRS = Pain Intensity Numeric Rating Scale; PP = per-protocol; RT-SFNSIQ= Rasch-transformed 13-item SFN Symptoms Inventory Questionnaire; SAE = serious adverse event; SF-36 = Short Form 36 Health Survey; SFN = small fiber neuropathy; SFN-RODS = Rasch-transformed SFN Overall Disability Scale.

Patients with small fiber neuropathy (SFN) commonly complain of length-dependent neuropathic pain symptoms due to dysfunction and degeneration of thinly myelinated $\mathrm{A} \delta$ and unmyelinated C fibers. ${ }^{1,2}$ Pain symptoms occur spontaneously (e.g., burning, deep, paroxysmal pain) and can be elicited by innocuous stimuli (e.g., light touch or pressure, warm and cold water). Different underlying systemic illnesses $^{3-11}$ and genetic diseases ${ }^{12-16}$ are associated with SFN. However, in 53\%, an underlying etiology remains unknown (idiopathic SFN [I-SFN]). Immunologic mechanisms have been speculated to contribute to patients with I-SFN because several immune-mediated disorders such as sarcoidosis, Sjogren disease, and systemic lupus erythematosus have shown some association with SFN. ${ }^{3,7,17-19}$ In addition, autoantibodies have been seen in patients with $\mathrm{SFN}^{20-23}$ inflammatory modifications in nerves have been noticed, ${ }^{24,25}$ raised proinflammatory cytokines potentially influence the pathophysiology of pain in $\mathrm{SFN}^{26}$ and peripheral and central glial-mediated neuroimmune activation has been reported in maintaining chronic pain. ${ }^{27}$

IV immunoglobulin (IVIG) is a successful, commonly used treatment for chronic immune-mediated polyneuropathies such as chronic inflammatory demyelinating polyneuropathy and multifocal motor neuropathy. ${ }^{28-30}$ Several open-label case series have reported IVIG to be efficacious in immunemediated $\mathrm{SFN},{ }^{31-37}$ and a recent retrospective study suggested IVIG as an effective treatment option for patients with SFN with autoimmune diseases or nonspecific blood test markers for autoimmunity. ${ }^{38}$ As a consequence, patients are frequently treated with this highly expensive drug, ${ }^{39}$ despite the lack of proven effectiveness for IVIG in I-SFN through controlled trials. We present the results of a double-blind randomized controlled trial evaluating the efficacy and safety of IVIG vs placebo in patients with I-SFN.

\section{Methods}

The IVIG in I-SFN study was a randomized, placebocontrolled, double-blind study. A complete report of the study design has been published earlier, ${ }^{40}$ and the outline is given below.

\section{Standard Protocol Approvals, Registrations, and Patient Consents}

The study was performed in accordance with the guidelines of the Declaration of Helsinki and International Conference on Harmonization Good Clinical Practice Guidelines. The study protocol was approved by the local Medical Ethics Committee. All patients in this trial gave written informed consent. This trial is registered with ClinicalTrials.gov (NCT02637700) and EudraCT (2015-002624-31).

\section{Study Design and Participants}

All consecutive patients, not from a prevalent pool, were included and treated at the SFN Center at the Maastricht University Medical Center+, the Netherlands, between July 2016 and March 2019, after giving written informed consent. Patients with I-SFN were eligible after meeting the international diagnostic criteria of SFN (i.e., typical SFN-related symptoms described mainly as a burning sensation, shooting pains, prickling or itching, predominantly in a lengthdependent pattern, with a minimum pain intensity score of $\geq 5$ on the Pain Intensity Numeric Rating Scale [PI-NRS], combined with a reduced distal intraepidermal nerve fiber density in skin biopsy, excluding large fiber involvement) and without an underlying etiology (such as diabetes mellitus, SCN9A/SCN10A/SCN11A mutations, hypothyroidism, vitamin $B_{12}$ deficiency, Fabry disease, Sjogren syndrome, sarcoidosis, and celiac disease), ${ }^{1,2,15,41}$ which was routinely tested in all patients before study entry. Exclusion criteria included prominent non-length-dependent pattern, predominant clinical picture of large nerve fiber involvement (i.e., weakness, loss of vibration sense, hypoflexia/areflexia, abnormal nerve conduction studies of tibial, peroneal, and sural nerves, including distal latency, amplitude, and conduction velocity using surface electrodes with standard placement), receiving IVIG treatment or any other immunomodulatory/immunosuppressive agents (e.g., steroids) within the 12 weeks before screening, and cardiac insufficiency (New York Heart Association class III/IV), cardiomyopathy, or significant cardiac dysrhythmia. The use of pain medication was allowed and registered if this remained stable in the 30 days before randomization. A change in dosage of (analgesic/antineuropathic) pain medication was not allowed throughout the study. Permissible medications were acetaminophen and nonsteroidal anti-inflammatory drugs such as ibuprofen, which were allowed during the trial as premedications for study drug infusions. A complete report of the inclusion and exclusion criteria was published previously. ${ }^{40}$

\section{Randomization and Masking}

Patients were randomly allocated in 2 groups (IVIG or placebo) using the ALEA randomization software, provided by FormsVision BV (Abcoude, the Netherlands) with the minimization technique. Patients were stratified according to age and sex. All participants, care providers, and study personnel, including those assessing outcomes, except for those in the 

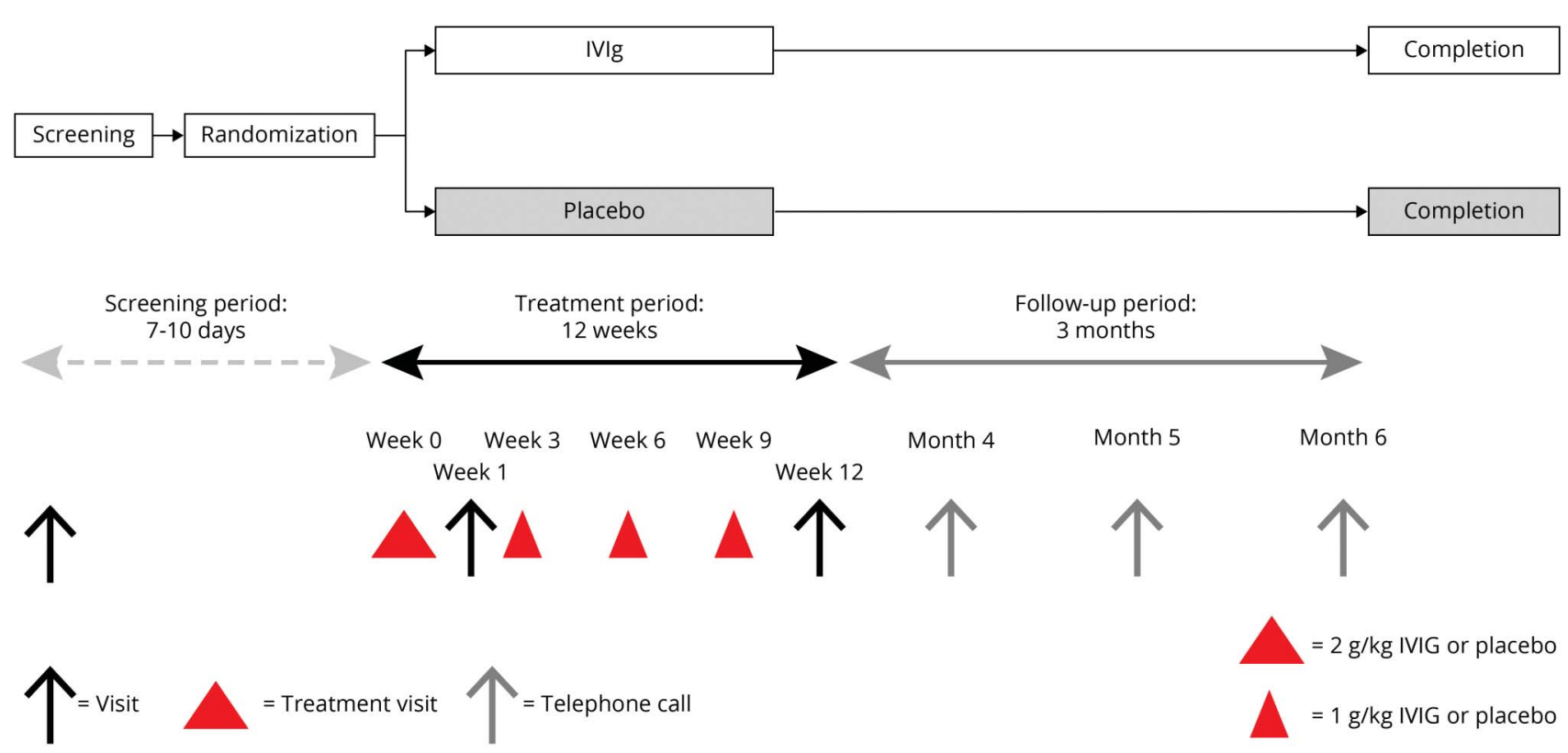

Red triangles represent the treatment visits. The first uploading treatment period was spread out over 2 consecutive days. The other treatment visits consisted of 1 day. IVIg = IV immunoglobulin.

pharmacy, were blinded for treatment assignment. IVIG and matching placebo were provided in ethylene vinyl acetate infusion bags with masking covers for IV administration. For optimal masking, orange infusion lines (B. Braun Cyto-Set Infusomat Space PZN10759513) were used. The blinding codes were intact during the complete study.

\section{Study Medication and Procedures}

The study medication was Gamunex (Grifols USA, LLC, Los Angeles, CA) $10 \%, 100 \mathrm{mg} / \mathrm{mL}$ solution for infusion of human normal immunoglobulin. Placebo was supplied as $0.9 \%$ saline. Figure 1 presents the study algorithm. ${ }^{40}$ The study started with a screening period (maximum 10 days). Eligible individuals subsequently entered the study treatment period (duration 12 weeks) and were randomized to conceive either IVIG with an uploading dose of $2 \mathrm{~g} / \mathrm{kg}$ body weight or placebo over 2 serial days, with a maximum dose of $80 \mathrm{~g}$ IVIG per infusion day. In addition, 3 infusions with a dose of $1 \mathrm{~g} / \mathrm{kg}$ body weight were dispensed with a 3-week interval. This regimen was chosen as previously applied in patients with chronic inflammatory demyelinating polyneuropathy for evaluating the IVIG efficacy. ${ }^{28} \mathrm{~A}$ final visit took place for all patients 3 weeks after the last infusion or after withdrawal for any reason during this period. After treatment completion, there was a 3-month-extension follow-up phase evaluating the possible long-term effect of IVIG on pain if any.

\section{Trial Outcomes}

The primary research question of this study was as follows: does IVIG significantly reduces pain compared to placebo for patients with painful I-SFN (Class I evidence)?
The primary efficacy outcome was the proportion of patients after the treatment period having at least a 1-point improvement on the 11-point PI-NRS ( $0=$ no pain, $10=$ worst imaginable pain) of their average pain compared to baseline, which is considered the minimum clinically important difference, according to unified rule of $0.5 \mathrm{SD}$ and the guidelines. ${ }^{42,43}$ Patients also completed a daily sleep interference scale (DSIS) (11-point numerical scale; $0=$ pain does not interfere with sleep, $10=$ pain completely interferes with sleep). ${ }^{44}$ Participants completed the PI-NRS and the DSIS 2 times per week in the morning and evening at scheduled time points. Additional questionnaires (patients' global impression of change [PGIC], ${ }^{42,43}$ the Raschtransformed 13-item SFN Symptoms Inventory Questionnaire [RT-SFN-SIQ], ${ }^{45}$ the Neuropathic Pain Scale [NPS], ${ }^{46}$ the Short Form 36 Health Survey [SF-36], and the Rasch-transformed SFN Overall Disability Scale [SFNRODS ], ${ }^{45}$ and the amount of pain relief [on a 5-point Likert scale $])^{47}$ were completed at each visit during the treatment period, during the telephone calls, and at the final follow-up visit. In addition, all pharmacologic pain treatments and pain-relieving activities were recorded. Safety evaluation characteristics included adverse events, laboratory tests, and vital signs.

Secondary efficacy outcomes were the proportion of patients having $\geq 2$-point average pain improvement compared to their baseline PI-NRS scores and changes in the mean, maximum, and average scores on the PI-NRS, NPS, DSIS, PGIC, Pain Relief, RT-SFN-SIQ, SFN-RODS, and SF-36. 


\section{Statistical Analyses}

The sample size of 24 participants per group was determined with an assumed response rate of $25 \%$ in the placebo group and $60 \%$ in the IVIG group, a 1 -sided $\alpha$ of $5 \%$, and $80 \%$ power between the 2 groups ( $\chi^{2}$ test), including the assumption of a dropout rate of $20 \%$ (6 patients). Therefore, the aim was to include in total 60 patients in the study. An independent statistician (S.M.J.v.K.) analyzed the results according to the intention-to-treat (ITT) protocol. ${ }^{40}$ For the primary ITT analysis for success, missing values were included as no success. Participants were analyzed for efficacy according to randomized treatment. For all questionnaires, the difference between baseline (screening period) and the end of the treatment period (outcomes of the 12th week) was calculated. To determine the baseline scores, all available data before randomization were taken. No data imputation was performed for missing observations. For the primary outcome, the proportion of patients with a decrease of at least 1 point was compared between the IVIG and placebo groups with a $\chi^{2}$ test. In addition, the proportion of $\geq 2$-point decrease was compared. When the PI-NRS score after 12 weeks was missing (because of dropout or missing diaries), patients were labeled as nonresponders.

The differences in DSIS, NPS, and SF-36 scores between baseline and 12 weeks of treatment were compared between the 2 groups. For these continuous outcome measures, the $t$ test was used. For the PGIC, the proportion of patients who were (very) much improved was compared to the proportion of patients with little or no improvement. For the RT-SFNSIQ and SFN-RODS, the proportions of patients with

Figure 2 Flowchart of the Trial

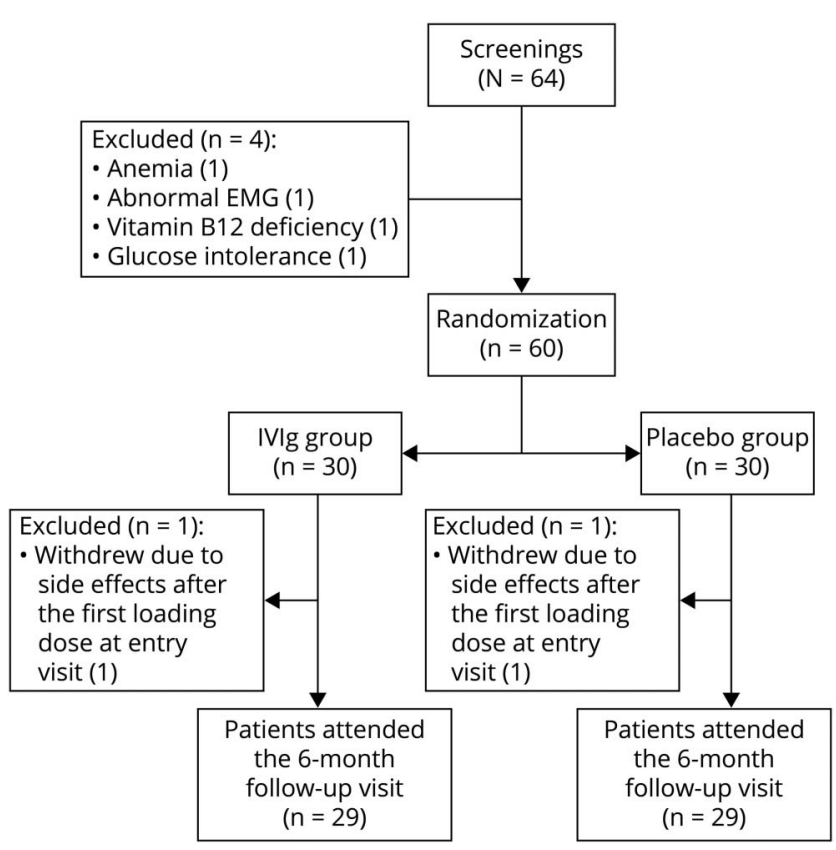

IVIg = IV immunoglobulin. significant deterioration, significant improvement, and no significant change were calculated and compared between the IVIG and placebo groups according to the distribution-based minimum clinically important difference-standard error method, with a meaningful change with a score $\geq 1.96$ standard error. ${ }^{48}$ For pain relief, the proportion of patients with no/slight relief was compared with the proportion of patients with moderate/good/complete relief. The $\chi^{2}$ test, or Fisher exact test when needed, was used to calculate the differences between the 2 treatment groups.

All the above-mentioned tests were repeated in the perprotocol (PP) analysis. Patients were included in the PP analysis if they had an available PI-NRS score at baseline and a 12-week postbaseline mean weekly pain PI-NRS score.

During the trial, the protocol was amended once to add a follow-up period, to remove the PGIC and Pain Relief questionnaires from the screening visit, to adjust the visit window, to add questions to patients' pain diary, and to adjust the infusion rates according to the protocol of the hospital.

\section{Data Availability}

Anonymized data not published in the article will be shared by request.

\section{Results}

\section{Patients}

After a thorough investigation, 64 patients met the inclusion and exclusion criteria, as shown in figure 2 . These patients were screened and gave informed consent for participating in the IVIG-SFN study. Four patients (6.3\%) were excluded due to anemia, abnormal EMG, vitamin $\mathrm{B}_{12}$ deficiency, and glucose intolerance $($ all $n=1)$.

Sixty patients were randomized, 30 patients (50\%) to IVIG and 30 to the placebo arm. Two patients (3.33\%) withdrew their participation due to side effects (nausea, anxiety, fatigue, headache, and diarrhea) after the first uploading study medication dose at entry visit ( 1 patient was randomized to the IVIG arm, the other to placebo). Ultimately, 29 patients who received IVIG and 29 patients who received placebo completed the study.

All 60 patients were randomly assigned to received IVIG or placebo, and 294 of the 300 scheduled volumes (>95\%) were actually dispensed. Patients endured a maximum volume of $240 \mathrm{~mL} / \mathrm{h}$ of the uploading dose and a maximal volume of 560 $\mathrm{mL} / \mathrm{h}$ for the additional infusions. Infusion time for the uploading dose was $\approx 5$ hours and for the additional infusions 3 hours.

\section{Baseline Characteristics}

Baseline characteristics are shown in table 1. Patients in the 2 groups were similar in demographic, clinical, and disease- 
Table 1 Baseline Characteristics of the Patients

\begin{tabular}{|c|c|c|}
\hline & $\begin{array}{l}\text { IVIG } \\
(n=30)\end{array}$ & $\begin{array}{l}\text { Placebo } \\
(n=30)\end{array}$ \\
\hline Age (at inclusion), mean (SD), y & $48.7(11.1)$ & $50.7(9.7)$ \\
\hline Male, n (\%) & $10(33.3)$ & $12(40.0)$ \\
\hline BMI, mean (SD), kg/m² & $29.1(5.0)$ & $27.7(5.1)$ \\
\hline $\begin{array}{l}\text { Duration of complaints SFN, median } \\
\text { (range), y }\end{array}$ & $\begin{array}{l}7.8 \\
(1.3-58.5)\end{array}$ & $\begin{array}{l}7.4 \\
(1.7-34.9)\end{array}$ \\
\hline White, n (\%) & $28(93.3)$ & $29(96.7)$ \\
\hline \multicolumn{3}{|l|}{ Presence of comorbidity, n (\%) } \\
\hline Hypertension & $6(20.0)$ & $4(13.3)$ \\
\hline Hypercholesterolemia & $7(23.3)$ & $5(16.7)$ \\
\hline Cardiac history & $1(3.3)$ & $1(3.3)$ \\
\hline Age at skin biopsy/diagnosis, mean (SD), y & $45.7(9.8)$ & $48.3(10.7)$ \\
\hline IENFD, mean (SD) ${ }^{a}$ & $3.2(1.7)$ & $3.1(1.5)$ \\
\hline TTT abnormal, n (\%) & $20(66.7)$ & $25(83.3)$ \\
\hline \multicolumn{3}{|l|}{ Diagnosis based on, n (\%) } \\
\hline Abnormal skin biopsy & $10(33.3)$ & $5(16.7)$ \\
\hline Abnormal skin biopsy and TTT deviation & $20(66.7 \%$ & $25(83.3)$ \\
\hline \multicolumn{3}{|l|}{ Presence of typical SFN symptoms, n (\%) } \\
\hline Burning feet & $27(90.0)$ & $26(86.7)$ \\
\hline Allodynia & $22(73.3)$ & $20(66.7)$ \\
\hline $\begin{array}{l}\text { Diminished pain or temperature } \\
\text { sensation }\end{array}$ & $21(70.0)$ & $21(70.0)$ \\
\hline Dry eyes or mouth & $26(86.7)$ & $26(86.7)$ \\
\hline Orthostatic dizziness & $21(70.0)$ & $12(40.0)$ \\
\hline Bowel disturbances & $19(63.3)$ & $22(73.3)$ \\
\hline Urinary disturbances & $18(60.0)$ & $18(60.0)$ \\
\hline Sweat changes & $20(66.7)$ & $24(80.0)$ \\
\hline $\begin{array}{l}\text { Visual accommodation problems or } \\
\text { blurred vision }\end{array}$ & $19(63.3)$ & $17(56.7)$ \\
\hline Hot flashes/palpitations & $21(70.0)$ & $16(53.3)$ \\
\hline $\begin{array}{l}\text { Impotence, diminished ejaculation or } \\
\text { lubrication }\end{array}$ & $11(36.7)$ & $13(43.3)$ \\
\hline $\begin{array}{l}\text { Total typical SFN symptoms, median } \\
\text { (range), } n\end{array}$ & $8(2-11)$ & $7(3-11)$ \\
\hline IgG before treatment, mean (SD) & $13.1(9.6)$ & $9.5(2.2)$ \\
\hline \multicolumn{3}{|l|}{ Use of (neuropathic) pain medication, $n(\%)$} \\
\hline \multicolumn{3}{|l|}{ Analgesics } \\
\hline Acetaminophen & $13(43.3)$ & $8(26.7)$ \\
\hline \multicolumn{3}{|l|}{ NSAID } \\
\hline Ibuprofen & $5(16.7)$ & $3(10.0)$ \\
\hline Diclofenac & $2(6.7)$ & $2(6.7)$ \\
\hline
\end{tabular}

Table 1 Baseline Characteristics of the Patients (continued)

\begin{tabular}{llc}
\hline & $\begin{array}{l}\text { IVIG } \\
(\mathbf{n}=\mathbf{3 0})\end{array}$ & $\begin{array}{l}\text { Placebo } \\
(\mathbf{n}=\mathbf{3 0})\end{array}$ \\
\hline TCA & $1(3.3)$ & $3(10.0)$ \\
\hline Amitriptyline & $1(3.3)$ & $1(3.3)$ \\
\hline Nortriptyline & & \\
\hline SNRI & $5(16.7)$ & $5(16.7)$ \\
\hline Duloxetine & $5(16.7)$ & $1(3.3)$ \\
\hline Anticonvulsant & $3(10.0)$ & $3(10.0)$ \\
\hline Gabapentin & $1(3.3)$ & $1(3.3)$ \\
\hline Pregabalin & & \\
\hline Carbamazepin & $4(13.3)$ & $1(3.3)$ \\
\hline Opioids & $2(6.7)$ & $1(3.3)$ \\
\hline Oxycodone & $0(0.0)$ & $1(3.3)$ \\
\hline Tramadol & & \\
\hline Local anesthetics & & \\
\hline Capsaicin creme & & \\
\hline
\end{tabular}

Abbreviations: $\mathrm{BMI}=$ body mass index; IENFD $=$ intraepidermal nerve fiber density; IgG = immunoglobulin G; IVIG = IV immunoglobulins; NSAID = nonsteroidal anti-inflammatory drugs; SFN = small fiber neuropathy; SNRI = selective serotonin and noradrenalin reuptake inhibitor; TCA = tricyclic antidepressants; TTT = temperature threshold testing

a Collected at the distal side of the right leg, $10 \mathrm{~cm}$ above the lateral malleolus; all patients had an abnormal IENFD (value below the fifth percentile of normal).

related characteristics at baseline. The baseline scores of the questionnaires are provided in table 2. For the ITT analyses, all 60 patients were used, and for the PP analysis, 10 patients were excluded (50 used; 24 in the IVIG group and 26 in the placebo group).

\section{Primary Outcome}

The primary outcome of the mean average pain scores based on the PI-NRS before and after treatment are shown in table 3: $40 \%$ of patients receiving IVIG and $30 \%$ of patients receiving placebo had at least a 1-point improvement in the average pain $(p=0.588$, odds ratio $1.56,95 \%$ confidence interval 0.53-4.53). The PP analysis and the 2-point decrease on the PI-NRS (ITT and PP analysis) also showed no significant differences.

\section{Secondary Outcomes}

Only a few of the secondary outcomes showed significant differences after 24 weeks (PI-NRS maximum night pain after 24 weeks $p=0.029$, DSIS $p=0.010$, NPS intense pain $p$ $=0.118$, NPS hot pain $p=0.031)$. SF-36 health change showed a significant difference after 12 weeks $(p=0.007)$; however, most secondary outcomes showed no significant difference between the effect of IVIG and the effect of placebo (table 3). 
Table 2 Baseline Scores of Patients

IVIG $(n=30) \quad$ Placebo $(n=30) \quad$ Total $(n=60)$

\begin{tabular}{|c|c|c|c|}
\hline \multicolumn{4}{|l|}{ PI-NRS score, median (range) } \\
\hline Mean day pain & $5.6(3.0-8.3)$ & $6.6(2.3-9.5)$ & $6.0(2.3-9.5)$ \\
\hline Mean night pain & $5.8(1.0-8.5)$ & $5.4(0.0-9.5)$ & $5.7(0.0-9.5)$ \\
\hline Mean average pain & $5.5(2.0-8.3)$ & $5.8(1.7-9.5)$ & $5.8(1.7-9.5)$ \\
\hline Maximum day pain & $7.0(3.7-10.0)$ & $7.8(3.3-10.0)$ & $7.2(3.3-10.0)$ \\
\hline Maximum night pain & $7.2(1.5-9.5)$ & $6.4(0.0-10.0)$ & $7.0(0.0-10.0)$ \\
\hline Maximum average pain & $7.0(3.0-9.8)$ & $7.0(2.2-10.0)$ & $7.0(2.2-10.0)$ \\
\hline DSIS score, median (range) & $5.5(1.0-8.7)$ & $5.8(0.0-9.0)$ & $5.5(0.0-9.0)$ \\
\hline SFN-SIQ score, median (range) & $17.5(6.0-35.0)$ & $17.0(6.0-35.0)$ & $17.0(6.0-35.0)$ \\
\hline SFN-RODS score, median (range) & $45.0(27.0-64.0)$ & $50.5(31.0-64.0)$ & $49.0(27.0-64.0)$ \\
\hline \multicolumn{4}{|l|}{ NPS score, median (range) } \\
\hline Intense & $7.0(4.0-10.0)$ & $7.0(2.0-10.0)$ & $7.0(2.0-10.0)$ \\
\hline Sharp & $8.0(0.0-10.0)$ & $7.0(0.0-10.0)$ & $7.5(0.0-10.0)$ \\
\hline Hot & $7.5(0.0-10.0)$ & $7.0(0.0-9.0)$ & $7.0(0.0-10.0)$ \\
\hline Dull & $5.0(0.0-10.0)$ & $4.5(0.0-9.0)$ & $5.0(0.0-10.0)$ \\
\hline Cold & $5.0(0.0-9.0)$ & $5.0(0.0-10.0)$ & $5.0(0.0-10.0)$ \\
\hline Sensitive & $5.5(0.0-10.0)$ & $6.0(0.0-10.0)$ & $6.0(0.0-10.0)$ \\
\hline Itchy & $1.5(0.0-10.0)$ & $5.0(0.0-9.0)$ & $4.0(0.0-10.0)$ \\
\hline Unpleasant & $7.5(4.0-10.0)$ & $7.0(3.0-10.0)$ & $7.0(3.0-10.0)$ \\
\hline Intense deep & $8.0(1.0-10.0)$ & $8.0(0.0-10.0)$ & $8.0(0.0-10.0)$ \\
\hline Intense surface & $6.0(2.0-9.0)$ & $6.0(2.0-10.0)$ & $6.0(2.0-10.0)$ \\
\hline \multicolumn{4}{|l|}{ SF-36 score, median (range) } \\
\hline Physical Functioning & $50.0(0.0-100.0)$ & $50.0(15.0-95.0)$ & $50.0(0.0-100.0)$ \\
\hline Social Functioning & $50.0(0.0-100.0)$ & $50.0(0.0-100.0)$ & $50.0(0.0-100.0)$ \\
\hline Role-Physical & $28.1(0.0-87.5)$ & $28.1(0.0-81.3)$ & $28.1(0.0-87.5)$ \\
\hline Role-Emotional & $83.3(0.0-100.0)$ & $75.0(8.3-100.0)$ & $79.2(0.0-100.0)$ \\
\hline Mental Health & $75.0(25.0-100.0)$ & $70.0(25.0-100.0)$ & $75.0(25.0-100.0)$ \\
\hline Vitality & $37.5(0.0-93.8)$ & $37.5(6.3-81.3)$ & $37.5(0.0-93.8)$ \\
\hline Bodily Pain & $32.7(0.0-79.6)$ & $35.7(10.2-69.4)$ & $33.7(0.0-79.6)$ \\
\hline General Health & $35.0(5.0-75.0)$ & $27.5(0.0-90.0)$ & $32.5(0.0-90.0)$ \\
\hline Health change & $25.0(0.0-75.0)$ & $25.0(0.0-100.0)$ & $25.0(0.0-100.0)$ \\
\hline
\end{tabular}

Abbreviations: DSIS = daily sleep interference scale; IVIG = IV immunoglobulin; NPS = Neuropathic Pain Scale; PI-NRS = Pain Intensity Numerical Rating Scale; SF-36 = generic Short-Form 36 Health Survey; SFN-RODS = Rasch-transformed Small Fiber Neuropathy Overall Disability Scale; SFN-SIQ = Small Fiber Neuropathy Symptoms Inventory Questionnaire.

After 12 weeks, 7 (24.1\%) patients in the IVIG group and 4 $(13.8 \%)$ in the placebo group were much or very much improved according to the PGIC $(p=0.505)$, and after 24 weeks, $4(13.8 \%)$ patients in the IVIG group vs $3(11.1 \%)$ in the placebo group $(p=1.000)$ were much or very much improved. The PP analysis showed similar results. In addition, no differences were found in autonomic symptoms, overall disability, and pain relief; the RT-SFN-SIQ SFN-RODS, and Pain Relief questionnaires, respectively, showed no significant differences between the 2 groups. 
Table 3 Primary and Secondary Outcomes

\begin{tabular}{|c|c|c|c|c|c|c|c|c|}
\hline \multirow[b]{2}{*}{ Primary outcome } & \multicolumn{4}{|l|}{ ITT } & \multicolumn{4}{|l|}{ PP } \\
\hline & \multicolumn{3}{|c|}{$\begin{array}{l}\text { Proportion of responders, IVIG vs placebo } \\
\text { (baseline vs } 3 \mathrm{mo} \text { ) }\end{array}$} & $\begin{array}{l}p \\
\text { Value }\end{array}$ & \multicolumn{3}{|c|}{$\begin{array}{l}\text { Proportion of responders, IVIG vs placebo } \\
\text { (baseline vs } 3 \mathrm{mo} \text { ) }\end{array}$} & $\begin{array}{l}p \\
\text { Value }\end{array}$ \\
\hline $\begin{array}{l}\geq 1 \text {-Point decrease in } \\
\text { average pain, } \mathrm{n}(\%)\end{array}$ & \multicolumn{3}{|c|}{12 (40) vs 9 (30) OR 1.56 (95\% Cl 0.54-4.63) } & 0.588 & \multicolumn{3}{|c|}{12 (50) vs 9 (34.6) OR 1.89 (95\% Cl 0.61-6.04) } & 0.415 \\
\hline$\geq 2$-Point decrease in & \multicolumn{3}{|c|}{7 (23.35) vs 5 (16.7) OR 1.52 (95\% Cl 0.43-5.78) } & 0.747 & \multicolumn{3}{|c|}{7 (29.2) vs 5 (19.2) OR 1.73 (95\% Cl 0.47-6.79) } & 0.624 \\
\hline & \multicolumn{3}{|l|}{ ITT } & & \multicolumn{3}{|l|}{ PP } & \\
\hline Secondary outcomes & $\begin{array}{l}\text { Difference, } \\
\text { baseline vs } 3 \text { mo, } \\
\text { IVIG vs placebo }\end{array}$ & $\begin{array}{l}p \\
\text { Value }\end{array}$ & $\begin{array}{l}\text { Difference, } \\
\text { baseline vs } 6 \text { mo, } \\
\text { IVIG vs placebo }\end{array}$ & $\begin{array}{l}p \\
\text { Value }\end{array}$ & $\begin{array}{l}\text { Difference, } \\
\text { baseline vs } 3 \text { mo, } \\
\text { IVIG vs placebo }\end{array}$ & $\begin{array}{l}p \\
\text { Value }\end{array}$ & $\begin{array}{l}\text { Difference, } \\
\text { baseline vs } 6 \text { mo, } \\
\text { IVIG vs placebo }\end{array}$ & $\begin{array}{l}p \\
\text { Value }\end{array}$ \\
\hline \multicolumn{9}{|l|}{ Continuous outcomes } \\
\hline \multicolumn{9}{|l|}{$\begin{array}{l}\text { PI-NRS score, mean } \\
\quad \text { (SEM) }\end{array}$} \\
\hline Mean day pain & $\begin{array}{l}-0.82(0.41) \text { vs } \\
-0.84(0.38)\end{array}$ & 0.973 & $\begin{array}{l}-0.72(0.26) \text { vs } \\
-0.46(0.26)\end{array}$ & 0.474 & $\begin{array}{l}-0.82(0.41) \text { vs } \\
-0.84(0.38)\end{array}$ & 0.973 & $\begin{array}{l}-0.82(0.27) \text { vs } \\
-0.51(0.28)\end{array}$ & 0.431 \\
\hline Mean night pain & $\begin{array}{l}-1.23(0.54) \text { vs } \\
-0.28(0.45)\end{array}$ & 0.185 & $\begin{array}{l}-1.08(0.45) \text { vs } \\
0.07(0.33)\end{array}$ & 0.046 & $\begin{array}{l}-1.23(0.54) \text { vs } \\
-0.28(0.45)\end{array}$ & 0.185 & $\begin{array}{l}-1.16(0.49) \text { vs } \\
-0.05(0.37)\end{array}$ & 0.075 \\
\hline Mean average pain & $\begin{array}{l}-0.89(0.38) \text { vs } \\
-0.51(0.36)\end{array}$ & 0.472 & $\begin{array}{l}-0.90(0.28) \text { vs } \\
-0.19(0.25)\end{array}$ & 0.067 & $\begin{array}{l}-0.89(0.38) \text { vs } \\
-0.51(0.36)\end{array}$ & 0.472 & $\begin{array}{l}-0.99(0.30) \text { vs } \\
-0.28(0.28)\end{array}$ & 0.086 \\
\hline DSIS score, mean (SEM) & $\begin{array}{l}-1.57(0.51) \text { vs } \\
-0.18(0.44)\end{array}$ & 0.045 & $\begin{array}{l}-1.17(0.38) \text { vs } \\
-0.43(0.27)\end{array}$ & 0.010 & $\begin{array}{l}-1.57(0.51) \text { vs } \\
-0.18(0.44)\end{array}$ & 0.045 & $\begin{array}{l}-1.64(0.53) \text { vs } \\
0.51(0.53)\end{array}$ & 0.006 \\
\hline \multicolumn{9}{|l|}{ NPS score, mean (SEM) } \\
\hline Intense & $\begin{array}{l}-1.9(0.47) \text { vs }-0.7 \\
(0.29)\end{array}$ & 0.033 & $\begin{array}{l}-1.72(0.38) \text { vs } \\
-0.43(0.27)\end{array}$ & 0.118 & $\begin{array}{l}-2.33(0.50) \text { vs } \\
-0.77(0.30)\end{array}$ & 0.011 & $\begin{array}{l}-0.46(0.41) \text { vs } \\
-0.56(0.26)\end{array}$ & 0.073 \\
\hline Sharp & $\begin{array}{l}-1.4(0.62) \text { vs }-0.6 \\
(0.43)\end{array}$ & 0.260 & $\begin{array}{l}-0.31(0.57) \text { vs } \\
-0.46(0.37)\end{array}$ & 0.821 & $\begin{array}{l}-1.92(0.71) \text { vs } \\
-0.46(0.44)\end{array}$ & 0.089 & $\begin{array}{l}-0.75(0.63) \text { vs } \\
-0.56(0.38)\end{array}$ & 0.797 \\
\hline Hot & $\begin{array}{l}-1.6(0.55) \text { vs } 0.2 \\
(0.44)\end{array}$ & 0.017 & $\begin{array}{l}-1.38(0.51) \text { vs } \\
0.18(0.48)\end{array}$ & 0.031 & $\begin{array}{l}-1.75(0.63) \text { vs } \\
0.15(0.48)\end{array}$ & 0.021 & $\begin{array}{l}-1.29(0.58) \text { vs } \\
0.20(0.52)\end{array}$ & 0.062 \\
\hline Dull & $\begin{array}{l}-1.8(0.70) \text { vs }-0.8 \\
(0.62)\end{array}$ & 0.273 & $\begin{array}{l}-1.07(0.84) \text { vs } \\
0.14(0.57)\end{array}$ & 0.237 & $\begin{array}{l}-1.50(0.80) \text { vs } \\
-0.96(0.67)\end{array}$ & 0.608 & $\begin{array}{l}-0.63(0.95) \text { vs } \\
0.16(0.59)\end{array}$ & 0.488 \\
\hline Cold & $\begin{array}{l}-1.4(0.62) \text { vs }-0.7 \\
(0.53)\end{array}$ & 0.354 & $\begin{array}{l}-1.86(0.66) \text { vs } \\
-0.18(0.64)\end{array}$ & 0.072 & $\begin{array}{l}-1.58(0.57) \text { vs } \\
-0.46(0.50)\end{array}$ & 0.146 & $\begin{array}{l}-1.83(0.61) \text { vs } \\
-0.28(0.63)\end{array}$ & 0.085 \\
\hline Sensitive & $\begin{array}{l}-1.9(0.53) \text { vs }-0.9 \\
(0.38)\end{array}$ & 0.108 & $\begin{array}{l}-0.28(0.53) \text { vs } \\
-0.64(0.47)\end{array}$ & 0.608 & $\begin{array}{l}-1.96(0.63) \text { vs } \\
-0.73(0.39)\end{array}$ & 0.107 & $\begin{array}{l}-0.29(0.64) \text { vs } \\
-0.60(0.51)\end{array}$ & 0.708 \\
\hline Itchy & $\begin{array}{l}-0.6(0.39) \text { vs }-1.0 \\
(0.61)\end{array}$ & 0.604 & $\begin{array}{l}-0.48(0.51) \text { vs } \\
-0.61(0.55)\end{array}$ & 0.868 & $\begin{array}{l}-0.75(0.42) \text { vs } \\
-1.15(0.57)\end{array}$ & 0.573 & $\begin{array}{l}-0.46(0.51) \text { vs } \\
-0.76(0.50)\end{array}$ & 0.674 \\
\hline Unpleasant & $\begin{array}{l}-1.7(0.48) \text { vs }-0.6 \\
(0.31)\end{array}$ & 0.052 & $\begin{array}{l}-0.93(0.42) \text { vs } \\
-0.46(0.34)\end{array}$ & 0.392 & $\begin{array}{l}-2.08(0.51) \text { vs } \\
-0.62(0.34)\end{array}$ & 0.022 & $\begin{array}{l}-1.25(0.39) \text { vs } \\
-0.44(0.37)\end{array}$ & 0.142 \\
\hline Intense deep & $\begin{array}{l}-1.5(0.48) \text { vs }-1.6 \\
(0.57)\end{array}$ & 0.889 & $\begin{array}{l}-0.76(0.38) \text { vs } \\
-0.39(0.50)\end{array}$ & 0.565 & $\begin{array}{l}-1.75(0.55) \text { vs } \\
-1.27(0.58)\end{array}$ & 0.548 & $\begin{array}{l}-0.88(0.44) \text { vs } \\
-0.16(0.53)\end{array}$ & 0.307 \\
\hline Intense surface & $\begin{array}{l}-0.9(0.52) \text { vs }-0.3 \\
(0.44)\end{array}$ & 0.365 & $\begin{array}{l}-0.86(0.49) \text { vs } \\
0.25(0.44)\end{array}$ & 0.097 & $\begin{array}{l}-1.42(0.54) \text { vs } \\
-0.69(0.42)\end{array}$ & 0.295 & $\begin{array}{l}-1.22(0.52) \text { vs } \\
-0.12(0.41)\end{array}$ & 0.106 \\
\hline \multicolumn{9}{|l|}{$\begin{array}{l}\text { SF-36 score, mean } \\
\text { (SEM) }\end{array}$} \\
\hline Physical Functioning & $\begin{array}{l}8.4(3.55) \text { vs } 1.2 \\
(2.23)\end{array}$ & 0.090 & $\begin{array}{l}4.5(4.61) \text { vs } 2.9 \\
(3.07)\end{array}$ & 0.773 & $\begin{array}{l}12.3(3.81) \text { vs }(2.11 \\
(2.27)\end{array}$ & 0.027 & $\begin{array}{l}10.22(4.28) \text { vs } \\
4.60(3.13)\end{array}$ & 0.296 \\
\hline Social Functioning & $\begin{array}{l}5.6(4.41) \text { vs } 6.9 \\
(3.60)\end{array}$ & 0.821 & $\begin{array}{l}4.5(5.58) \text { vs } 1.3 \\
(4.13)\end{array}$ & 0.655 & $\begin{array}{l}8.90(4.76) \text { vs } 6.25 \\
(3.81)\end{array}$ & 0.553 & $\begin{array}{l}9.24(5.84) \text { vs } 1.50 \\
(4.64)\end{array}$ & 0.305 \\
\hline Role-Physical & $\begin{array}{l}13.6(4.09) \text { vs } 0.6 \\
(3.83)\end{array}$ & 0.025 & $\begin{array}{l}6.5(4.45) \text { vs }(3.3 \\
(4.22)\end{array}$ & 0.612 & $\begin{array}{l}13.28(4.78) \text { vs } \\
2.16(4.07)\end{array}$ & 0.083 & $\begin{array}{l}8.70(5.30) \text { vs } 4.25 \\
(4.59)\end{array}$ & 0.529 \\
\hline
\end{tabular}


Table 3 Primary and Secondary Outcomes (continued)

\begin{tabular}{|c|c|c|c|c|c|c|c|c|}
\hline \multirow[b]{2}{*}{ Secondary outcomes } & \multicolumn{4}{|l|}{ ITT } & \multicolumn{4}{|l|}{ PP } \\
\hline & $\begin{array}{l}\text { Difference, } \\
\text { baseline vs } 3 \text { mo, } \\
\text { IVIG vs placebo }\end{array}$ & $\begin{array}{l}p \\
\text { Value }\end{array}$ & $\begin{array}{l}\text { Difference, } \\
\text { baseline vs } 6 \text { mo, } \\
\text { IVIG vs placebo }\end{array}$ & $\begin{array}{l}p \\
\text { Value }\end{array}$ & $\begin{array}{l}\text { Difference, } \\
\text { baseline vs } 3 \text { mo, } \\
\text { IVIG vs placebo }\end{array}$ & $\begin{array}{l}p \\
\text { Value }\end{array}$ & $\begin{array}{l}\text { Difference, } \\
\text { baseline vs } 6 \text { mo, } \\
\text { IVIG vs placebo }\end{array}$ & $\begin{array}{l}p \\
\text { Value }\end{array}$ \\
\hline Role-Emotional & $\begin{array}{l}1.4(4.96) \text { vs }-3.2 \\
(3.93)\end{array}$ & 0.471 & $\begin{array}{l}-6.8(5.22) \text { vs }-2.4 \\
(5.07)\end{array}$ & 0.542 & $\begin{array}{l}4.17(3.40) \text { vs } \\
-4.81(4.24)\end{array}$ & 0.106 & $\begin{array}{l}-4.35(4.25) \text { vs } \\
-3.00(5.63)\end{array}$ & 0.849 \\
\hline Mental Health & $\begin{array}{l}2.9(2.39) \text { vs } 1.2 \\
(2.19)\end{array}$ & 0.596 & $\begin{array}{l}-1.3(3.07) \text { vs } 2.0 \\
(2.54)\end{array}$ & 0.423 & $\begin{array}{l}3.33(2.57) \text { vs } 1.15 \\
(2.36)\end{array}$ & 0.535 & $\begin{array}{l}-1.96(3.57) \mathrm{vs} \\
2.20(2.65)\end{array}$ & 0.355 \\
\hline Vitality & $\begin{array}{l}7.5(3.96) \text { vs }-0.4 \\
(1.81)\end{array}$ & 0.075 & $\begin{array}{l}4.7(3.47) \text { vs } 0.0 \\
(1.90)\end{array}$ & 0.243 & $\begin{array}{l}8.59(3.59) \text { vs } \\
-0.24(1.89)\end{array}$ & 0.036 & $\begin{array}{l}7.07(3.78) \text { vs } 0.25 \\
(1.99)\end{array}$ & 0.120 \\
\hline Bodily pain & $\begin{array}{l}12.0(3.95) \text { vs } 5.9 \\
(3.03)\end{array}$ & 0.229 & $\begin{array}{l}7.7(4.19) \text { vs } 2.7 \\
(3.32)\end{array}$ & 0.358 & $\begin{array}{l}15.31(4.24) \text { vs } \\
5.73(3.37)\end{array}$ & 0.084 & $\begin{array}{l}11.36(4.46) \text { vs } \\
3.02(3.65)\end{array}$ & 0.156 \\
\hline General health & $\begin{array}{l}9.0(2.95) \text { vs } 5.7 \\
(2.59)\end{array}$ & 0.408 & $\begin{array}{l}3.8(2.32) \text { vs } 3.4 \\
(2.60)\end{array}$ & 0.919 & $\begin{array}{l}10.21(3.41) \text { vs } \\
5.58(2.81)\end{array}$ & 0.300 & $\begin{array}{l}5.43(2.63) \text { vs } 3.00 \\
(2.89)\end{array}$ & 0.536 \\
\hline Health change & $\begin{array}{l}34.5(6.73) \text { vs } 11.2 \\
(4.90)\end{array}$ & 0.007 & $\begin{array}{l}20.5(5.76) \text { vs } 8.9 \\
(4.31)\end{array}$ & 0.113 & $\begin{array}{l}34.38(6.87) \text { vs } \\
12.50(5.42)\end{array}$ & 0.016 & $\begin{array}{l}21.74(6.35) \text { vs } \\
11.00(4.58)\end{array}$ & 0.178 \\
\hline
\end{tabular}

\begin{tabular}{|c|c|c|c|c|c|c|c|c|}
\hline \multicolumn{9}{|l|}{ Discrete outcomes } \\
\hline PGIC, n (\%) & & 0.505 & & 1.000 & & 0.490 & & 1.000 \\
\hline Very much improved & 7 (24.1) vs 4 (14) & & 4 (14) vs 3 (11) & & 6 (25) vs 4 (15) & & 4 (17) vs 3 (13) & \\
\hline $\begin{array}{l}\text { Little or not } \\
\text { improved }\end{array}$ & 22 (75.9) vs 25 (86) & & 25 (86) vs 26 (89) & & 18 (75) vs 22 (85) & & 20 (83) vs 23 (87) & \\
\hline RT-SFN-SIQ, n (\%) & & 0.194 & & 0.3574 & & 0.340 & & 0.355 \\
\hline $\begin{array}{l}\text { Significant } \\
\text { deterioration (1) }\end{array}$ & $0(0)$ vs $0(0)$ & & $0(0)$ vs $2(7)$ & & $0(0)$ vs $0(0)$ & & $0(0)$ vs $2(8)$ & \\
\hline $\begin{array}{l}\text { No significant } \\
\text { change (0) }\end{array}$ & 24 (83) vs 28 (97) & & 26 (90) vs 25 (89) & & 21 (88) vs 25 (96) & & 21 (88) vs 22 (88) & \\
\hline $\begin{array}{l}\text { Significant } \\
\text { improvement }(-1)\end{array}$ & 5 (17) vs 1 (3) & & 3 (10) vs 1 (4) & & 3 (13) vs 1 (4) & & 3 (13) vs 1 (4) & \\
\hline SFN-RODS, n (\%) & & 0.378 & & 0.378 & & 0.122 & & 0.375 \\
\hline $\begin{array}{l}\text { Significant } \\
\text { deterioration (-1) }\end{array}$ & 2 (7) vs 4 (14) & & 6 (21) vs 5 (19) & & $0(0)$ vs $3(12)$ & & 3 (13) vs 4(17) & \\
\hline $\begin{array}{l}\text { No significant } \\
\text { change }(0)\end{array}$ & 23 (79) vs 24 (83) & & 17 (59) vs 19 (73) & & 20 (83) vs 22 (85) & & 15 (63) vs 18 (75) & \\
\hline $\begin{array}{l}\text { Significant } \\
\text { improvement (1) }\end{array}$ & 4 (14) vs 1 (3) & & $6(21)$ vs $2(8)$ & & 4 (17) vs 1 (4) & & $6(25)$ vs $2(8)$ & \\
\hline Pain-relief, n (\%) & & 0.214 & & 0.254 & & 0.348 & & 0.245 \\
\hline No/slight relief & 19 (79) vs 17 (94) & & 23 (79) vs 25 (93) & & 16 (80) vs 16 (94) & & 18 (75) vs 22 (92) & \\
\hline $\begin{array}{l}\text { Moderate/Good/ } \\
\text { complete relief }\end{array}$ & $5(21)$ vs $1(6)$ & & $6(21)$ vs $2(7)$ & & $4(20)$ vs $1(6)$ & & $6(25)$ vs $2(8)$ & \\
\hline
\end{tabular}

Abbreviations: CI = confidence interval; DSIS = daily sleep interference scale; IVIG = IV immunoglobulin; ITT = intention-to-treat; NPS = Neuropathic Pain Scale; $\mathrm{OR}=$ odds ratio; PGIC = patients' global impression of change; PI-NRS = Pain Intensity Numerical Rating Scale; PP = per-protocol; RT-SFN-SIQ = Raschtransformed 13-item Small Fiber Neuropathy Symptoms Inventory Questionnaire; SF-36 = generic Short-Form 36 Health Survey; SFN-RODS = Raschtransformed Small Fiber Neuropathy Overall Disability Scale; SFN-SIQ = Small Fiber Neuropathy Symptom Inventory Questionnaire.

\section{Safety}

Thirty patients in the IVIG group (100\%) had at least 1 adverse event (table 4) compared to 29 in the placebo group (96.7\%). In total, 6 serious adverse events (SAEs) were reported, including aorta coarctation repair, gastrointestinal hemorrhage, headache, hospitalization (multiple complaints), pulmonary embolism, and suicide attempt. Only 1 SAE (aorta coarctation repair) occurred in the placebo group; the others occurred in the IVIG group. The gastrointestinal hemorrhage was diagnosed before randomization. Only 1 SAE in the IVIG group, hospitalization (multiple complaints), could have been caused by the use of 
Table 4 Adverse Events

\begin{tabular}{|c|c|c|c|}
\hline & IVIG $(n=30), n(\%)$ & Placebo $(n=30), n(\%)$ & $p$ Value \\
\hline Patients with at least 1 adverse event & $30(100)$ & $29(96.7)$ & 1.000 \\
\hline \multicolumn{4}{|l|}{ All SAES } \\
\hline Aorta coarctation repair & $0(0.0)$ & $1(3.3)$ & 1.000 \\
\hline Gastrointestinal hemorrhage & $1(3.3)$ & $0(0.0)$ & 1.000 \\
\hline Headache & $1(3.3)$ & $0(0.0)$ & 1.000 \\
\hline Hospitalization & $1(3.3)$ & $0(0.0)$ & 1.000 \\
\hline Pulmonary embolism & $1(3.3)$ & $0(0.0)$ & 1.000 \\
\hline Suicide attempt & $1(3.3)$ & $0(0.0)$ & 1.000 \\
\hline \multicolumn{4}{|l|}{ Most common adverse events ${ }^{a}$} \\
\hline Headache & $30(100)$ & $17(56.7)$ & $<0.001$ \\
\hline Nausea & $19(63.3)$ & $7(23.3)$ & 0.004 \\
\hline Other pain & $18(60.0)$ & $14(46.7)$ & 0.438 \\
\hline Vomiting & $11(36.7)$ & $0(0.0)$ & 0.001 \\
\hline Chills & $9(30.0)$ & $3(10.0)$ & 0.104 \\
\hline Dizziness & $8(26.7)$ & $9(30.0)$ & 1.000 \\
\hline Rash & $8(26.7)$ & $0(0.0)$ & 0.005 \\
\hline Fatigue & $7(23.3)$ & $11(36.7)$ & 0.399 \\
\hline Influenza & $7(23.3)$ & $2(6.7)$ & 0.146 \\
\hline Arthralgia & $4(13.3)$ & $6(20.0)$ & 0.731 \\
\hline Hyperhidrosis & $4(13.3)$ & $3(10.0)$ & 1.000 \\
\hline Pyrexia & $4(13.3)$ & $4(13.3)$ & 1.000 \\
\hline Diarrhea & $3(10.0)$ & $0(0.0)$ & 0.237 \\
\hline Myalgia & $2(6.7)$ & $2(6.7)$ & 1.000 \\
\hline Nasopharyngitis & $1(3.3)$ & $2(6.7)$ & 1.000 \\
\hline Vision blurred & $1(3.3)$ & $3(10.0)$ & 0.612 \\
\hline Cough & $1(3.3)$ & $2(6.7)$ & 1.000 \\
\hline Migraine & $0(0.0)$ & $2(6.7)$ & 0.492 \\
\hline
\end{tabular}

Abbreviations: IVIG = IV immunoglobulin; SAE = serious adverse events

${ }^{a}$ Only the adverse events that occurred in at least $5 \%$ of the patients are given.

IVIG because it occurred 2 weeks after the first loading dose. The other 3 SAEs seemed not to have been caused by the use of IVIG because they occurred in the follow-up phase (2-4 weeks after the last infusion).

The adverse events that occurred in at least $5 \%$ of the patients are shown in table 4 . All patients in the IVIG group (100\%) had headache compared to $56.7 \%$ in the placebo group. Nausea (63.3\% vs $23.3 \%$ ), vomiting ( $36.7 \%$ vs $0.0 \%$ ), and rash $(26.7 \%$ vs $0.0 \%)$ occurred significantly more frequently in the IVIG group compared to the placebo group, respectively.
Because patients were allowed to use their own medications during the study (see table 1 for neuropathic pain medication use at baseline), the differences between patients with and without the use of neuropathic pain medication were investigated regarding the primary outcome (table 5). No significant differences between the groups were found.

\section{Discussion}

This is the first randomized placebo-controlled study that investigated the efficacy of IVIG in patients with painful 
Table 5 Neuropathic Pain Medication During the Study

\begin{tabular}{|c|c|c|c|c|}
\hline Patients using neuropathic pain medication & $\begin{array}{l}\text { IVIG group, } \\
\text { Responders vs gonresponders } \\
\text { n (\%) }\end{array}$ & $p$ Value & $\begin{array}{l}\text { Placebo group, } \\
\text { Responders vs gonresponders } \\
\text { n (\%) }\end{array}$ & $p$ Value \\
\hline \multicolumn{5}{|l|}{ ITT population } \\
\hline 21-Point decrease in pain & 8 (66.7) vs 11 (61.1) & 1.000 & 7 (77.8) vs $11(52.4)$ & 0.249 \\
\hline$\geq 2$-Point decrease in pain & 5 (71.4) vs 14 (60.9) & 1.000 & $4(80.0)$ vs $14(56.0)$ & 0.622 \\
\hline \multicolumn{5}{|l|}{ PP population } \\
\hline$\geq 1$-Point decrease in pain & $8(66.7)$ vs $6(50.0)$ & 0.679 & 7 (77.8) vs 9 (52.9) & 0.399 \\
\hline$\geq 2$-Point decrease in pain & 5 (71.4) vs 9 (52.9) & 0.653 & $4(80.0)$ vs 12 (57.1) & 0.617 \\
\hline
\end{tabular}

Abbreviations: ITT = intention-to-treat; IVIG = IV immunoglobulin; PP = per-protocol.

I-SFN. The results of this study showed no significant effect of IVIG compared to placebo in patients with painful I-SFN. No significant differences were found in 1-point and 2-point responders on pain, PGIC, overall disability, autonomic symptoms, and pain relief. Statistically significant differences were found in maximum night pain, daily sleep interference, intense and hot pain, and some domains of the quality of life; however they were not in a consistent pattern and were not considered to be of meaningful clinical relevance in the treatment goal of this highly expensive drug. Six SAEs occurred, 5 of them in the IVIG group, and all patients in the IVIG group experienced at least 1 adverse event, of which headache, nausea, vomiting, and rash occurred significantly more frequently compared to the placebo group. All of these are adverse events of IVIG that are known to occur at rates $>10 \%{ }^{28}$ It can therefore be concluded that IVIG has no place in the standard treatment of painful I-SFN.

The results of this study are in contrast to open-label case series, which included many patients with an immunemediated underlying condition, ${ }^{31-33,35-37}$ and a retrospective study suggesting the efficacy of IVIG in SFN. ${ }^{31,32,38}$ However, according to those reports, patients with SFN, without or without a clear autoimmune condition, are increasingly being treated with IVIG. ${ }^{39}$ It is important to point out that certain patients with immune-confirmed SFN etiologies may benefit from IVIG treatment, and it is crucial for effective neuropathic pain treatment to perform detailed upfront testing on autoimmune diseases in patients with SFN. ${ }^{15}$ Nevertheless, future trials in these setting are necessary to determine the value of IVIG treatment in this specific cohort.

Our study has some limitations. First, the investigated cohort was relatively small and limited to patients with painful I-SFN. This outlined cohort was chosen because we aimed to demonstrate a possible proof of concept, which could be used for future studies involving larger groups of patients diagnosed with painful I-SFN. The sample size of 60 patients was calculated on the basis of the assumption that the IVIG-treated group would have a response rate of $\approx 60 \%$ based on the
Initiative on Methods, Measurement, and Pain Assessment in Clinical Trials criteria ${ }^{43}$ and the placebo-treated group would have a response rate of $\approx 25 \%$ according to a meta-analysis of the placebo effect in pain studies. ${ }^{49}$ Second, the study was powered to find a difference between $60 \%$ response rate in the IVIG group and $25 \%$ in the placebo group. The observed score proportions were $40 \%$ and $30 \%$, respectively, which were below our expectations and thereby underline our findings that IVIG is not a proven treatment for patients with painful I-SFN. Even though the 10\% difference in response rate could be a statistically significant difference when the sample size of the study was much larger, this is not a clinically relevant difference. Third, the exclusion criteria did not describe fibromyalgia syndrome or complex regional pain syndrome, so unexpectedly, these patients could have been involved in the investigated cohort. However, before being diagnosed with SFN, some patients of our study with unexplained complaints could be (falsely) labeled as having fibromyalgia syndrome or complex regional pain syndrome because the clinical picture may show similarities, but an abnormal skin biopsy has now been found labeling them as having SFN. Fourth, our study was limited to those with painful I-SFN, so statements about IVIG to treat pain in those with autoimmune diseases underlying SFN are limited. Our results discourage the use of IVIG in this setting until randomized controlled trials are completed. Finally, although patients with psychiatric disorders were not excluded from the study, in some cases, the hospital psychiatry department was consulted before inclusion. For future IVIG treatment studies with larger groups of patients with painful I-SFN, it is recommend to assess and evaluate psychiatric comorbidity beforehand.

Some might argue that although the study was statistically negative, more (40\%) in the IVIG-treated group responded than in the placebo-treated group (30\%), and they would still want to treat patients to find responders. Thus, 10 patients would need to be treated with IVIG to find 1 nonplacebo responder (for the 1-point decrease in average pain) because, of the 4 of 10 who will respond to IVIG, 3 would be expected 
to be a placebo responder. For the 2-point decrease in pain, 15 patients should be treated to find 1 IVIG responder. The cost per gram of IVIG in the Netherlands is $€ 81.64 .{ }^{50}$ For a $75-\mathrm{kg}$ patient, assuming a $1-\mathrm{g} / \mathrm{kg}$ dose, the cost for this study of 5 infusions in 3 months was $€ 30,615$. Thus, finding 1 IVIG responder with a 1-point decrease in pain will cost $€ 306,150$, and finding 1 IVIG responder with a 2-point decrease in pain will cost $€ 459,225$ for 3 months only (without even taking into account the costs for the inpatient admission or home nurse visit, infusion pump, disposables, etc). Furthermore, all patients in the IVIG group experienced adverse events, including 6 SAEs, which carry significant risks and can further increase the costs associated with IVIG use.

This randomized controlled trial showed that IVIG has no significant effect on pain in patients with painful I-SFN and therefore has no role in the treatment of patients with painful I-SFN.

\section{Study Funding}

This study is funded by the Grifols Investigator-Sponsored Research Program and Lamepro B.V.

\section{Disclosure}

M. Geerts, Bianca T.A. de Greef, Maurice Sopacua, and Sander M.J. van Kuijk have nothing to disclose. Janneke G.J. Hoeijmakers reports a grant from the Prinses Beatrix Spierfonds (W.OK17-09), outside the submitted work. C.G. Faber reports grants from European Union's Horizon 2020 research and innovation programme Marie Sklodowska-Curie grant for PAIN-Net, Molecule-to-Man Pain Network (grant 721841), grants from Prinses Beatrix Spierfonds (W.OR1525), grants from Grifols and Lamepro for a trial on IVIG in SFN, and other from steering committtees/advisory board for studies in SFN of Biogen/Convergence and Vertex, outside the submitted work. I.S.J. Merkies reports grants and nonfinancial support from Grifols and grants from Lamepro, during the conduct of the study, as well as other from participation in steering committees of the Talecris ICE Study, CSL Behring, LFB, Novartis, Octapharma, Biotest and UCB, outside the submitted work. Go to Neurology.org/ $\mathrm{N}$ for full disclosures.

\section{Publication History}

Received by Neurology August 12, 2020. Accepted in final form February 24, 2021.

Appendix Authors

\begin{tabular}{|c|c|c|}
\hline Name & Location & Contribution \\
\hline $\begin{array}{l}\text { Margot } \\
\text { Geerts, MSc }\end{array}$ & $\begin{array}{l}\text { Department of Neurology, } \\
\text { School of Mental Health } \\
\text { and Neuroscience, } \\
\text { Maastricht University } \\
\text { Medical Center+, the } \\
\text { Netherlands }\end{array}$ & $\begin{array}{l}\text { Screening of patients, data } \\
\text { collection, data analysis, } \\
\text { and manuscript writing }\end{array}$ \\
\hline
\end{tabular}

\section{Appendix (continued)}

\begin{tabular}{|c|c|c|}
\hline Name & Location & Contribution \\
\hline $\begin{array}{l}\text { Bianca T.A. de } \\
\text { Greef, MD, } \\
\text { PhD }\end{array}$ & $\begin{array}{l}\text { Department of Neurology, } \\
\text { School of Mental Health } \\
\text { and Neuroscience, } \\
\text { Maastricht University } \\
\text { Medical Center+, the } \\
\text { Netherlands }\end{array}$ & $\begin{array}{l}\text { Screening of patients, data } \\
\text { collection, data analysis, } \\
\text { and manuscript writing }\end{array}$ \\
\hline
\end{tabular}

\begin{tabular}{lll}
\hline Maurice & $\begin{array}{l}\text { Department of Neurology, } \\
\text { Sopacua, MD }\end{array}$ & $\begin{array}{l}\text { Scheening of patients, data } \\
\text { collection and manuscript } \\
\text { and Neuroscience, } \\
\text { Maastricht University }\end{array}$ \\
& Medical Center+, the & \\
& Netherlands; Department \\
& of Rehabilitation \\
& Adelante/Maastricht \\
& University Medical \\
& Center+, the Netherlands
\end{tabular}

\begin{tabular}{lll}
\hline Sander M.J. & Department of Clinical & Data analysis, statistical \\
van Kuijk, & Epidemiology and Medical & $\begin{array}{l}\text { analysis, and manuscript } \\
\text { PhD }\end{array}$ \\
& Technology Assessment, & writing \\
& Maastricht University & \\
& Medical Center+, the & \\
& Netherlands &
\end{tabular}

\begin{tabular}{|c|c|c|}
\hline $\begin{array}{l}\text { Janneke G.J. } \\
\text { Hoeijmakers, } \\
\text { MD, PhD }\end{array}$ & $\begin{array}{l}\text { Department of Neurology, } \\
\text { School of Mental Health } \\
\text { and Neuroscience, } \\
\text { Maastricht University } \\
\text { Medical Center+, the } \\
\text { Netherlands }\end{array}$ & $\begin{array}{l}\text { Conception, design, data } \\
\text { collection, data analysis, } \\
\text { and manuscript writing }\end{array}$ \\
\hline $\begin{array}{l}\text { Catharina G. } \\
\text { Faber, MD, } \\
\text { PhD }\end{array}$ & $\begin{array}{l}\text { Department of Neurology, } \\
\text { School of Mental Health } \\
\text { and Neuroscience, } \\
\text { Maastricht University } \\
\text { Medical Center+, the } \\
\text { Netherlands }\end{array}$ & $\begin{array}{l}\text { Conception, design, data } \\
\text { collection, data analysis, } \\
\text { and manuscript writing }\end{array}$ \\
\hline $\begin{array}{l}\text { Ingemar S.J. } \\
\text { Merkies, MD, } \\
\text { PhD }\end{array}$ & $\begin{array}{l}\text { Department of Neurology, } \\
\text { School of Mental Health } \\
\text { and Neuroscience, } \\
\text { Maastricht University } \\
\text { Medical Center+, the } \\
\text { Netherlands; Department } \\
\text { of Neurology, Curaçao } \\
\text { Medical Center, } \\
\text { Willemstad }\end{array}$ & $\begin{array}{l}\text { Conception, design, data } \\
\text { collection, data analysis, } \\
\text { and manuscript writing }\end{array}$ \\
\hline
\end{tabular}

\section{References}

1. Hoeijmakers JG, Faber CG, Lauria G, Merkies IS, Waxman SG. Small-fibre neuropathies-advances in diagnosis, pathophysiology and management. Nat Rev Neurol 2012;8:369-379.

2. Cazzato D, Lauria G. Small fibre neuropathy. Curr Opin Neurol 2017;30:490-499.

3. Hoitsma E, Marziniak M, Faber CG, et al. Small fibre neuropathy in sarcoidosis. Lancet 2002;359:2085-2086.

4. Sumner CJ, Sheth S, Griffin JW, Cornblath DR, Polydefkis M. The spectrum of neuropathy in diabetes and impaired glucose tolerance. Neurology 2003;60:108-111.

5. Brannagan TH III, Hays AP, Chin SS, et al. Small-fiber neuropathy/neuronopathy associated with celiac disease: skin biopsy findings. Arch Neurol 2005;62:1574-1578.

6. Gondim FA, Brannagan TH III, Sander HW, Chin RL, Latov N. Peripheral neuropathy in patients with inflammatory bowel disease. Brain 2005;128:867-879.

7. Mori K, Iijima M, Koike $\mathrm{H}$, et al. The wide spectrum of clinical manifestations in Sjogren's syndrome-associated neuropathy. Brain 2005;128:2518-2534.

8. Goransson LG, Tjensvoll AB, Herigstad A, Mellgren SI, Omdal R. Small-diameter nerve fiber neuropathy in systemic lupus erythematosus. Arch Neurol 2006;63: 401-404.

9. Orstavik K, Norheim I, Jorum E. Pain and small-fiber neuropathy in patients with hypothyroidism. Neurology 2006;67:786-791.

10. Zhou L, Kitch DW, Evans SR, et al. Correlates of epidermal nerve fiber densities in HIV-associated distal sensory polyneuropathy. Neurology 2007;68:2113-2119.

11. Gorson KC, Herrmann DN, Thiagarajan R, et al. Non-length dependent small fibre neuropathy/ganglionopathy. J Neurol Neurosurg Psychiatry 2008;79:163-169.

12. Stogbauer F, Young P, Kuhlenbaumer G, et al. Autosomal dominant burning feet syndrome. J Neurol Neurosurg Psychiatry 1999;67:78-81. 
13. Faber CG, Hoeijmakers JG, Ahn HS, et al. Gain of function $\mathrm{Na}_{\mathrm{V}} 1.7$ mutations in idiopathic small fiber neuropathy. Ann Neurol 2012;71:26-39.

14. Faber CG, Lauria G, Merkies IS, et al. Gain-of-function Nav1.8 mutations in painful neuropathy. Proc Natl Acad Sci USA 2012;109:19444-19449.

15. de Greef BTA, Hoeijmakers JGJ, Gorissen-Brouwers CML, Geerts M, Faber CG, Merkies ISJ. Associated conditions in small fiber neuropathy: a large cohort study and review of the literature. Eur J Neurol 2018;25:348-355.

16. Huang J, Han C, Estacion M, et al. Gain-of-function mutations in sodium channel $\mathrm{Na}(\mathrm{v}) 1.9$ in painful neuropathy. Brain 2014;137:1627-1642.

17. Bakkers M, Merkies IS, Lauria G, et al. Intraepidermal nerve fiber density and its application in sarcoidosis. Neurology 2009;73:1142-1148.

18. Goransson LG, Herigstad A, Tjensvoll AB, Harboe E, Mellgren SI, Omdal R. Peripheral neuropathy in primary Sjogren syndrome: a population-based study. Arch Neurol 2006;63:1612-1615.

19. Goransson LG, Brun JG, Harboe E, Mellgren SI, Omdal R. Intraepidermal nerve fiber densities in chronic inflammatory autoimmune diseases. Arch Neurol 2006;63: 1410-1413.

20. Chamberlain JL, Pittock SJ, Oprescu AM, et al. Peripherin-IgG association with neurologic and endocrine autoimmunity. J Autoimmun 2010;34:469-477.

21. Ferrari S, Morbin M, Nobile-Orazio E, et al. Antisulfatide polyneuropathy: antibodymediated complement attack on peripheral myelin. Acta Neuropathol 1998;96: 569-574.

22. Dabby R, Weimer LH, Hays AP, Olarte M, Latov N. Antisulfatide antibodies in neuropathy: clinical and electrophysiologic correlates. Neurology 2000;54: $1448-1452$.

23. Levine TD, Kafaie J, Zeidman LA, et al. Cryptogenic small-fiber neuropathies: serum autoantibody binding to trisulfated heparan disaccharide and fibroblast growth factor receptor-3. Muscle Nerve 2019;61:512-515.

24. Zafrir B, Zimmerman M, Fellig Y, Naparstek Y, Reichman N, Flatau E. Small fiber neuropathy due to isolated vasculitis of the peripheral nervous system. Isr Med Assoc J 2004;6:183-184.

25. Kelkar P, McDermott WR, Parry GJ. Sensory-predominant, painful, idiopathic neuropathy: inflammatory changes in sural nerves. Muscle Nerve 2002;26:413-416.

26. Uceyler N, Kafke W, Riediger N, et al. Elevated proinflammatory cytokine expression in affected skin in small fiber neuropathy. Neurology 2010;74:1806-1813.

27. Goebel A. Immunoglobulin responsive chronic pain. J Clin Immunol 2010;30(suppl 1):S103-S108.

28. Hughes RA, Donofrio P, Bril V, et al. Intravenous immune globulin (10\% caprylatechromatography purified) for the treatment of chronic inflammatory demyelinating polyradiculoneuropathy (ICE study): a randomised placebo-controlled trial. Lancet Neurol 2008;7:136-144

29. van Schaik IN, Bouche P, Illa I, et al. European Federation of Neurological Societies/ Peripheral Nerve Society guideline on management of multifocal motor neuropathy. Eur J Neurol 2006;13:802-808.

30. Eftimov F, Winer JB, Vermeulen M, de Haan R, van Schaik IN. Intravenous immunoglobulin for chronic inflammatory demyelinating polyradiculoneuropathy. Cochrane Database Syst Rev 2013:CD001797.

31. Parambil JG, Tavee JO, Zhou L, Pearson KS, Culver DA. Efficacy of intravenous immunoglobulin for small fiber neuropathy associated with sarcoidosis. Respir Med 2011;105:101-105
32. Wakasugi D, Kato T, Gono T, et al. Extreme efficacy of intravenous immunoglobulin therapy for severe burning pain in a patient with small fiber neuropathy associated with primary Sjogren's syndrome. Mod Rheumatol 2009;19:437-440.

33. Gaillet A, Champion K, Lefaucheur JP, Trout H, Bergmann JF, Sene D. Intravenous immunoglobulin efficacy for primary Sjogren's Syndrome associated small fiber neuropathy. Autoimmun Rev 2019;18:102387.

34. Makonahalli R, Seneviratne J, Seneviratne U. Acute small fiber neuropathy following mycoplasma infection: a rare variant of Guillain-Barre syndrome. J Clin Neuromuscul Dis 2014;15:147-151.

35. Tavee JO, Karwa K, Ahmed Z, Thompson N, Parambil J, Culver DA. Sarcoidosisassociated small fiber neuropathy in a large cohort: clinical aspects and response to IVIG and anti-TNF alpha treatment. Respir Med 2017;126:135-138.

36. Souayah N, Chin RL, Brannagan TH, et al. Effect of intravenous immunoglobulin on cerebellar ataxia and neuropathic pain associated with celiac disease. Eur J Neurol $2008 ; 15: 1300-1303$.

37. Schofield JR, Chemali KR. How we treat autoimmune small fiber polyneuropathy with immunoglobulin therapy. Eur Neurol 2018;80:304-310.

38. Liu X, Treister R, Lang M, Oaklander AL. IVIG for apparently autoimmune smallfiber polyneuropathy: first analysis of efficacy and safety. Ther Adv Neurol Disord 2018;11:1756285617744484

39. Oaklander AL, Nolano M. Scientific advances in and clinical approaches to small-fiber polyneuropathy: a review. JAMA Neurol Epub 2019 Sep 9.

40. de Greef BT, Geerts M, Hoeijmakers JG, Faber CG, Merkies IS. Intravenous immunoglobulin therapy for small fiber neuropathy: study protocol for a randomized controlled trial. Trials 2016;17:330.

41. Tesfaye S, Boulton AJ, Dyck PJ, et al. Diabetic neuropathies: update on definitions, diagnostic criteria, estimation of severity, and treatments. Diabetes Care 2010;33:2285-2293.

42. Farrar JT. What is clinically meaningful: outcome measures in pain clinical trials. Clin J Pain 2000;16:S106-S112.

43. Dworkin RH, Turk DC, Wyrwich KW, et al. Interpreting the clinical importance of treatment outcomes in chronic pain clinical trials: IMMPACT recommendations. J Pain 2008;9:105-121.

44. Vernon MK, Brandenburg NA, Alvir JM, Griesing T, Revicki DA. Reliability, validity, and responsiveness of the daily sleep interference scale among diabetic peripheral neuropathy and postherpetic neuralgia patients. J Pain Symptom Manage 2008;36:54-68.

45. Brouwer BA, Bakkers M, Hoeijmakers JG, Faber CG, Merkies IS. Improving assessment in small fiber neuropathy. J Peripher Nerv Syst 2015;20:333-340.

46. Galer BS, Jensen MP. Development and preliminary validation of a pain measure specific to neuropathic pain: the Neuropathic Pain Scale. Neurology 1997;48:332-338.

47. Aaronson NK, Muller M, Cohen PD, et al. Translation, validation, and norming of the Dutch language version of the SF-36 Health Survey in community and chronic disease populations. J Clin Epidemiol 1998;51:1055-1068.

48. Draak THP, de Greef BTA, Faber CG, Merkies ISJ; PeriNomS Study Group. The minimum clinically important difference: which direction to take. Eur J Neurol 2019, 26:850-855.

49. McQuay H, Carroll D, Moore A. Variation in the placebo effect in randomised controlled trials of analgesics: all is as blind as it seems. Pain 1996;64:331-335.

50. Farmacotherapeutisch Kompas-Gamunex-last visit. 2019. Available at: farmacotherapeutischkompas.nl/bladeren/preparaatteksten/n/normaal_immunoglobuline iv . Accessed May 19, 2015 


\section{Neurology}

\section{Intravenous Immunoglobulin Therapy in Patients With Painful Idiopathic Small Fiber Neuropathy}

Margot Geerts, Bianca T.A. de Greef, Maurice Sopacua, et al.

Neurology 2021;96;e2534-e2545 Published Online before print March 25, 2021

DOI 10.1212/WNL.0000000000011919

\section{This information is current as of March 25, 2021}

\section{Updated Information \& Services}

References

Citations

Subspecialty Collections

Permissions \& Licensing

Reprints including high resolution figures, can be found at: http://n.neurology.org/content/96/20/e2534.full

This article cites 47 articles, 11 of which you can access for free at: http://n.neurology.org/content/96/20/e2534.full\#ref-list-1

This article has been cited by 12 HighWire-hosted articles: http://n.neurology.org/content/96/20/e2534.full\#\#otherarticles

This article, along with others on similar topics, appears in the following collection(s):

Class I

http://n.neurology.org/cgi/collection/class_1

Clinical trials Randomized controlled (C̄ONSORT agreement)

http://n.neurology.org/cgi/collection/clinical_trials_randomized_contro lled_consort_agreement

Neuropathic pain

http://n.neurology.org/cgi/collection/neuropathic_pain

Information about reproducing this article in parts (figures,tables) or in its entirety can be found online at:

http://www.neurology.org/about/about_the_journal\#permissions

Information about ordering reprints can be found online:

http://n.neurology.org/subscribers/advertise

Neurology ${ }^{\circledR}$ is the official journal of the American Academy of Neurology. Published continuously since 1951, it is now a weekly with 48 issues per year. Copyright Copyright ( 2021 The Author(s). Published by Wolters Kluwer Health, Inc. on behalf of the American Academy of Neurology.. All rights reserved. Print ISSN: 0028-3878. Online ISSN: 1526-632X.

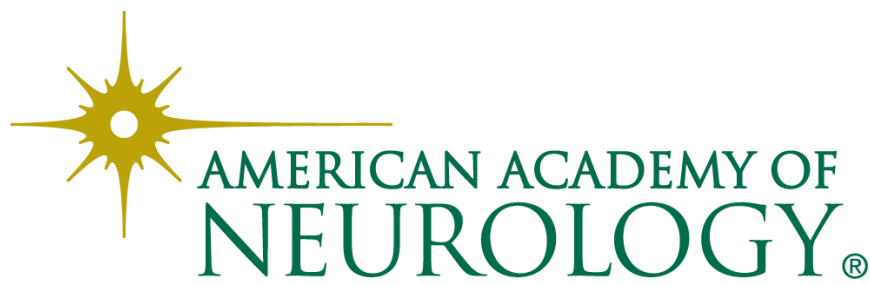

